\title{
Outgrowth by Fin Motor Axons in Wildtype and a Finless Mutant of the Japanese Medaka Fish
}

\author{
Hitoshi OKamoto, ${ }^{*, 1}$ and John Y. KuWAD ${ }^{*,+} \neq$ \\ *Deparłment of Biology, + Neuroscience Program, and $\ddagger$ Institute of Gerontology, University of Michigan, Ann Arbor, Michigan 48109 \\ Accepted March 26, 1991
}

\begin{abstract}
The outgrowth of motor axons to the developing pectoral fin of the Japanese medaka fish (Oryzias latipes) was investigated both in wildtype embryos and in the pectoral finless $(p l)$ mutants in which adults are missing pectoral fins. Late in embryogenesis the pectoral fin is a simple limb which contains two antagonist muscles which are innervated by presumptive motor neurons from the first four spinal segments (S1-4). The pectoral fin develops from a fin bud located in S1 and S2 centered on the border between S1 and S2 and, as with other limbs, one of the earliest signs of differentiation is the apical ectodermal ridge (AER). By the time the AER is well formed the growth cones of the presumptive motor neurons have reached the base of the fin bud and formed a plexus by extending toward the fin bud upon emergence from the spinal cord. This is especially evident on the ventral surface of the metamerically arranged axial muscles. For example, growth cones from $\mathrm{S} 2$ extend in a diagonal direction (both anterior and lateral) towards the fin bud. One hypothesis which can account for the pattern of motor outgrowth is that growth cones are attracted to the base of the fin bud, perhaps via a long distance cue. This hypothesis was tested by examining outgrowth of segmental nerves in $p l$ embryos in which the fin buds arrest early in development following the initial appearance of the AER. In $p l$, nerves from S1-4 converged to form a plexus at the base of the abnormal fin bud, but the pattern of outgrowth varied from wildtype in a way consistent with a diminished eapacity of the fin bud to attract segmental nerves to it. 1991 Academic Press, Inc.
\end{abstract}

\section{INTRODUCTION}

Vertebrate limbs are innervated by nerves from a defined set of spinal segments which converge to form a plexus at the base of the limb. The factors which influence the outgrowth of limb nerves to developing limbs have been investigated in a variety of animals. These studies have implicated possible long distance cues (Hamburger, 1929, 1939; Detwiler, 1934, 1936), inhibitory actions of sclerotome cells (Tosney and Oakley, 1990), local cues found at the base of the limb bud (Landmesser, 1988), barrier functions (Tosney and Landmesser, 1985), and death of mesenchyme cells preceding the motor growth cones (Tosney et al., 1988) for guidance to the limb. Although it is quite clear that a variety of mechanisms work in concert to achieve appropriate outgrow th to the limb, the evidence for some of these mechanisms is inconclusive. This is partly due to the complexity of the environment growth cones must navigate to reach the limb, complexity of the limb itself, and difficulty of manipulating limb buds without affecting the substrates leading to the limb bud. Furthermore, descriptions of outgrowth by limb motor axons have, for the most part, emphasized events occurring

\footnotetext{
${ }^{1}$ Present address: National Institute for Basic Binlogy, 38 Nishigonaka, Myodaiji-cho, Okazaki, Aichi 444, Japan.
}

within and beyond the plexus rather than leading up to plexus formation.

For several reasons the early pectoral fin is an attractive system for the analysis of mechanisms which guide fin motor growth cones. (1) It is a simple limb containing the abductor and adductor muscles and cartilage and a levator muscle which develops later (Sperry, 1950). (2) The clarity of the fish embryo and the ease with which growth cones and axons can be labeled with antibodies or dyes simplifies the analysis of pathfinding because axonal outgrowth can be studied in whole mounts. (3) Fin buds can be mechanically manipulated (Okamoto and Kuwada, 1991). (4) A mutation which affects fin development (Tomita, 1982) is available for analysis of outgrowth by fin motor axons in an altered environment.

We have taken advantage of these features to analyze outgrowth by fin motor axons, with an emphasis on pathfinding from the spinal cord to the base of the fin bud, in the embryo of the Japanese medaka fish. This paper describes the development of the pectoral fin and the outgrowth pattern of the fin motor growth cones in wildtype embryos and in mutant embryos with defective fin development. The companion paper (Okamoto and Kuwada, 1991) analyzes outgrowth by motor axons following fin bud ablations and following transplantation of fin buds to ectopic sites. 


\section{MATERIALS AND METHODS}

\section{Embryos}

Japanese medaka embryos were collected from a breeding colony, raised, and staged as previously reported (Kirchen and West, 1976; Kuwada, 1986). Hours of development refer to number of hours postfertilization at $26^{\circ} \mathrm{C}$.

\section{Histology}

Embryos were embedded in paraffin, sectioned, and stained with hemotoxylin and eosin or embedded in plastic and sectioned (Kuwada, 1986).

\section{Labeling Segmental Nerves and Motor Neurons}

Peripheral nerves were labeled by application of a monoclonal antibody against acetylated $\alpha$-tubulin (Piperno and Fuller, 1985) to whole mounted embryos using previously described procedures (Patel et al., 1989). This antibody has been demonstrated to effectively label many if not all axons in the fish embryo (Chitnis and Kuwada, 1990; Bernhardt et al., 1990; Kuwada et al., 1990).

Neurons were anterogradely or retrogradely labeled by application of the lipophilic dyes, diI and diO (Honig and IIume, 1986). Embryos were fixed overnight at $4^{\circ} \mathrm{C}$ in $4 \%$ paraformaldehyde in $75 \mathrm{~m} M$ phosphate buffer ( $\mathrm{pH}$ 7.4) and then washed briefly in buffer. The yolk sacks were removed and the embryos were embedded in agar (2\% agar in $75 \mathrm{mM}$ phosphate buffer) on a glass slide. DiI or diO $(0.25 \%$ in dimethylformamide) was pressure injected using a Picospritzer (General Valve Corp.) into spinal cords, ventral roots, or fin buds from microclectrodes with tips broken off. In some cases labeled neurons were marked with a brown reaction product by photooxidation in diaminobenzidine (Maranto, 1982). Some embryos in which axons were labeled by the acetylated tubulin antibody or by diI and photooxidation were embedded in plastic, cut into $1 \mu \mathrm{m}$ sections, and stained with toluidine blue by standard procedures.

Neurons were also back-labeled by application of horseradish peroxidase (HRP) to live embryos. Embryos were anesthetized with $0.03 \%$ tricaine and immobilized in 2\% agar mixed in Yamamoto's saline (Yamamoto, 1975). Recrystalized HRP was applied to the pectoral fin bud with a sharpened tungsten needle. Embryos were freed from the agar and kept in Yamamoto's saline for approximately $1 \mathrm{hr}$ then fixed in $2 \%$ paraformaldehyde, $1 \%$ glutaraldehyde, $1 \%$ dimethylsulfoxide in $75 \mathrm{~m} M$ phosphate buffer for $1 \mathrm{hr}$. Following several washes in phosphate buffer, embryos were immersed in $20 \%$ sucrose in phosphate buffer overnight and then embedded in $10 \%$ gelatin and $20 \%$ sucrose in phosphate buffer. Gelatin blocks containing embryos were fixed in $4 \%$ paraformaldehyde for $1 \mathrm{hr}$ and washed in $20 \%$ sucrose in phosphate buffer. Blocks were frozen and sectioned $(25 \mu \mathrm{m})$ with a cryostat using standard procedures. Sections were collected on slides and labeled neurons were visualized by the Hanker-Yates reaction (Hanker et al., 1977). The total number of back-labeled motor neurons were determined using the method described by Abercrombie (1946).

\section{Histograms and Polar Plots}

The amount of branching by segmental nerves as the nerves were extending out toward the fin bud on the ventral surface of the axial muscles was quantified by examining 35 embryos in which the segmental nerves were labeled with the acetylated tubulin antibody. Histograms were constructed from 35 embryos between 58 and $64 \mathrm{hr}$ of development. A labeled process was counted as a nerve branch if its tip was at least $3 \mu \mathrm{m}$ from its branch point. Polar plots were made to indicate the directionality of nerve branches for nerves from S1-3 on the ventral surface of the axial muscles from the same 35 embryos used above. The tips of segmental nerve branches were plotted with the origin of the polar coordinates corresponding to the point where the segmental nerve emerged onto the ventral surface of the axial muscle block in that segment and the medial border of the axial muscles aligned with the base of the polar plot. Data were collected into $10^{\circ}$ sectors.

\section{RESULTS}

\section{Late Embryonic Pectoral Fin and Nerves}

The pectoral fin of the 10-day-old embryo of the Japanese medaka fish, which is just about to hatch, is located in the first and second spinal segments (S1 and S2) with its attachment to the main body trunk located near the $\mathrm{S} 1 / \mathrm{S} 2$ border $(n=9)$. It contains two prominent muscles, the adductor (extensor) and abductor (flexor), separated by cartilage (not shown). Labeling axons with an antibody against acetylated $\alpha$-tubulin (Piperno and Fuller, 1985; Chitnis and Kuwada, 1990; Bernhardt et al., 1990) demonstrated that the fin is innervated by nerves which project from the plexus found at the base of the fin $(n=7)$. Two sets of similar nerves (one for the adductor muscle and one for the abductor muscle) run posteriorly from the anterior portion of the plexus, one on either side of the cartilage (Fig. 1). These nerves branch to give a series of parallel branches which run distally into the muscles.

The plexus is made up by the convergence of axons from nerves from S1-4 (Fig. 1B). Before entering the plexus the S1 nerve branches, one branch entering the 

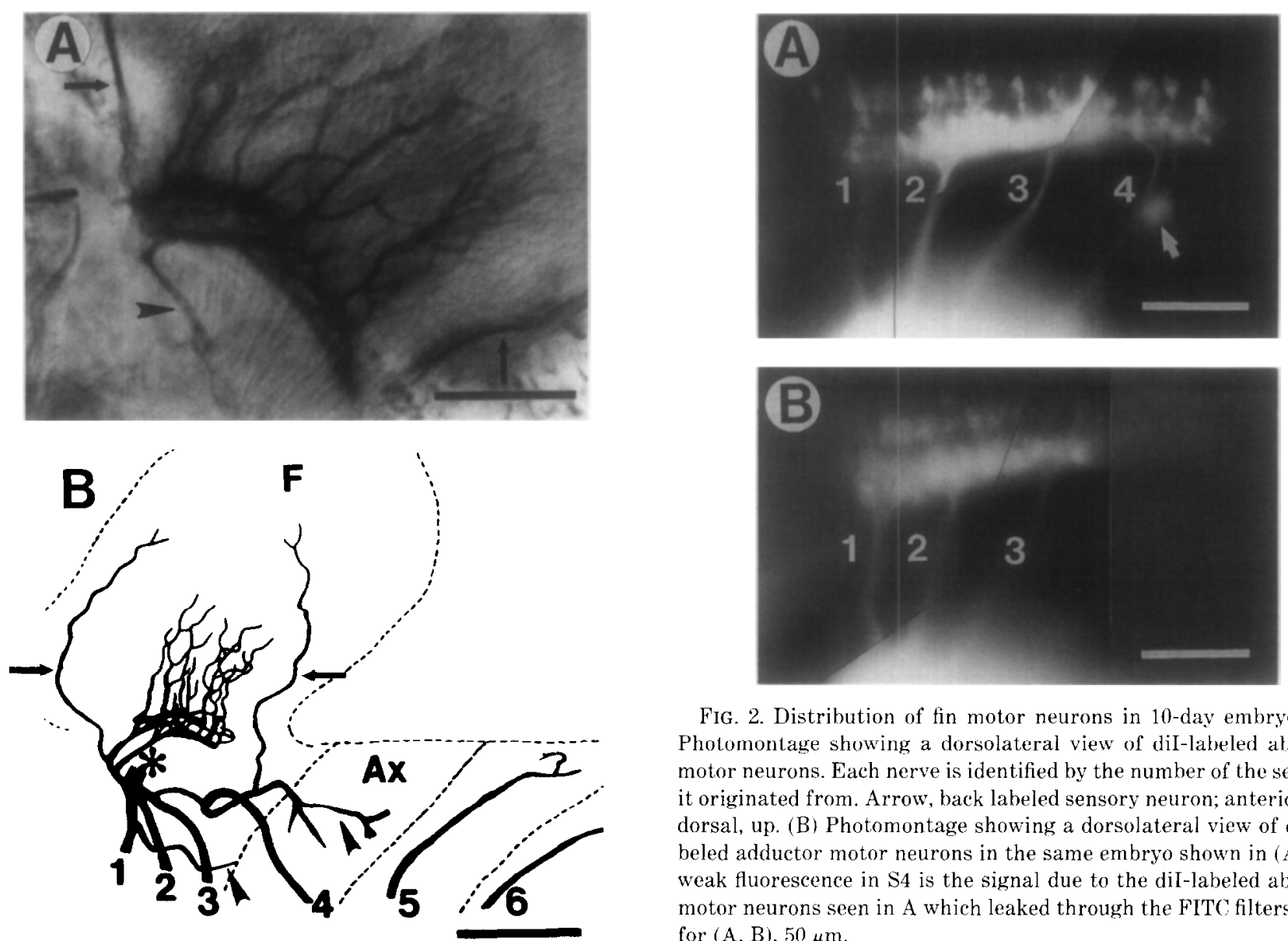

FIG. 2. Distribution of fin motor neurons in 10-day embryos. (A) Photomontage showing a dorsolateral view of diI-labeled abductor motor neurons. Each nerve is identified by the number of the segment it originated from. Arrow, back labeled sensory neuron; anterior, left; dorsal, up. (B) Photomontage showing a dorsolateral view of diO-labeled adductor motor neurons in the same embryo shown in (A). The weak fluorescence in $\mathrm{S} 4$ is the signal due to the diI-labeled abductor motor neurons seen in A which leaked through the FITC filters. Scale for $(\mathrm{A}, \mathrm{B}), 50 \mu \mathrm{m}$.

FIG. 1. The pectoral fin and its innervation in 10-day-old embryos. (A) Side view of the nerve branches innervating the adductor museles. Axons were labeled with the acetylated tubulin antibody. Anterior, left; scale $50 \mu \mathrm{m}$. Putative sensory axons are indicated by arrows. A branch of S1 which turns away from the fin ventrally is denoted by an arrow head. (B) Camera lucida drawing of the plexus, nerve branches innervating the abductor muscle, and presumptive sensory branches from an acetylated tubulin-labeled embryo. For simplicity the nerve branches innervating the adductor muscles, which have similar trajectories, were not drawn. The proximal portion of the adductor nerve branch is designated by an asterisk. Putative sensory axons are indicated by arrows. The branches of $\mathrm{S1}$ and $\mathrm{S4}$ which turn away from the fin are denoted by arrowheads. The fin, F, was rotated to allow the fin nerves and the segmental nerves (each nerve is designated by the number of the segment it originated from) to be drawn. Scale, $100 \mu \mathrm{m}$.

plexus and the other running ventrally away from the fin to an, as yet, unknown destination. Similarly the S4 nerve branches just before entering the plexus with a thin branch entering the plexus and a thick one veering postcriorly and ventrally away from the plexus. Occasionally (1 of 7 embryos), the S5 nerve projects a very thin anterior branch that enters the plexus.

The distribution of motor neurons which innervated the pectoral fin muscles in 10-day embryos was determined by back-labeling them with HRP applied to the

fin ( $n=20$; not shown). The largest number of motor neurons back-labeled in one side of a spinal cord was 133 in S1-4. There is much overlap in the distribution pattern of the motor neurons which terminate in the adductor and abductor muscles as determined by labeling them by application of diI and diO to the two muscles ( $n$ $=4$; Fig. 2$)$ or diI into one of the muscles $(n=3)$ in 10-day embryos. Adductor motor neurons were found in $\mathrm{S} 1-3$ while abductor motor neurons were located in S1-4.

\section{Development of the Pectoral Fin}

The pectoral fin in the Japanese medaka fish develops in essentially the same pattern as in other fishes (Bouvet, 1978; Wood, 1982). By $60 \mathrm{hr}$ (late stage 26) the fin bud is detectable as a gentle swelling $(n=43)$ with a slightly thickened ectoderm but no other obvious morphological signs of differentiation (Fig. 3A). Ten hours later (late stage $27 ; n=9$ ) the ectoderm overlying the distal tip of the fin bud has clearly started to differentiate into a structure with a cleft which based on morphological criteria appears to be the apical ectodermal ridge (AER) (Fig. 3B). By 84 hr (stage $29 ; n=5$ ) the AER 
has enlargened, the cleft is very conspicuous, and the underlying mesenchyme has coalesced into three blocks of cells (Fig. 3C), which later will differentiate into the two muscles and cartilage. Due to the large yolk sack the fin bud is located on the yolk sack, lateral to the main trunk of the embryo, and is oriented so that its proximodistal axis is parallel to the dorsoventral axis of the embryo with its base sitting on the yolk sack throughout early embryogenesis (Fig. 3).

\section{Pattern of Outgrowth by Fin and Nonfin Segmental Nerves}

The pattern of nerve outgrowth was assessed by labeling axons with the acetylated tubulin antibody in whole-mounted embryos (Fig. 4). Motor axons emerge from the spinal cord and extend ventrally between the medial surface of the axial muscles and the notochord (data not shown). At approximately $56 \mathrm{hr}$ (stage 26) nerves from S1-3 reach the ventral surface of the axial muscles and begin to extend laterally. By $58-64 \mathrm{hr}$ the segmental nerves from S1-3 have extended laterally and branched on the ventral surface of the axial muscles $(n$ $=34$; Figs. $4 \mathrm{~A}$ and $4 \mathrm{~B}$; Figs. $5 \mathrm{~A}-\mathrm{C}$ ). Although there is considerable variability, $S 1$ branches are predominantly directed either parallel to the border between $\mathrm{S} 1$ and S2 or away from the base of the fin bud (Fig. 5D). The borders between the segmented axial muscles are slanted and run at an angle of approximately $30^{\circ}$ posteriorly from the medial/lateral axis. Therefore, the S1 branches which are parallel to the segment border are directed towards the fin bud. The S2 and S3 branches are predominantly directed in an anterior/lateral direction towards the fin bud (Figs. $5 \mathrm{E}$ and $5 \mathrm{~F}$ ). At this stage unlike nerves from $\mathbf{S} 2-3$, the $\mathbf{S} 4$ nerve is extending in parallel with the segmental borders of axial muscles and, therefore, initially away from rather than towards the base of the fin bud (Fig. 4A).

By $64 \mathrm{hr}$ (early stage $27 ; n=5$ ) branches of the S1 and S2 nerve have already extended into the fin bud and the S4 nerve has branched (Fig. 4B). Six hours later (late stage $27 ; n=7$ ) there is much less branching on the portion of the nerves running laterally (i.e., prior to the plexus) and nerves from S1-3 have just converged and begun to form a plexus at the base of the fin bud (Fig.

FIG. 3. Development of the pectoral fin bud. (A) Cross section of a 60-hr embryo showing the ectoderm and underlying mesenchyme of the early fin bud, F, overlying the yolk. S, spinal cord; N, notochord; Ax, axial muscle. (B) Fin bud of a 70-hr embryo. AER, apical ectodermal ridge; $P$, pronephros. (C) Fin bud of a 84 -hr embryo. The fin bud mesenchyme has coalesced into a central core of cells which presumably will give rise to cartilage surrounded by cells which presumably will give rise to muscle. Scale for $(A-C), 20 \mu \mathrm{m}$.
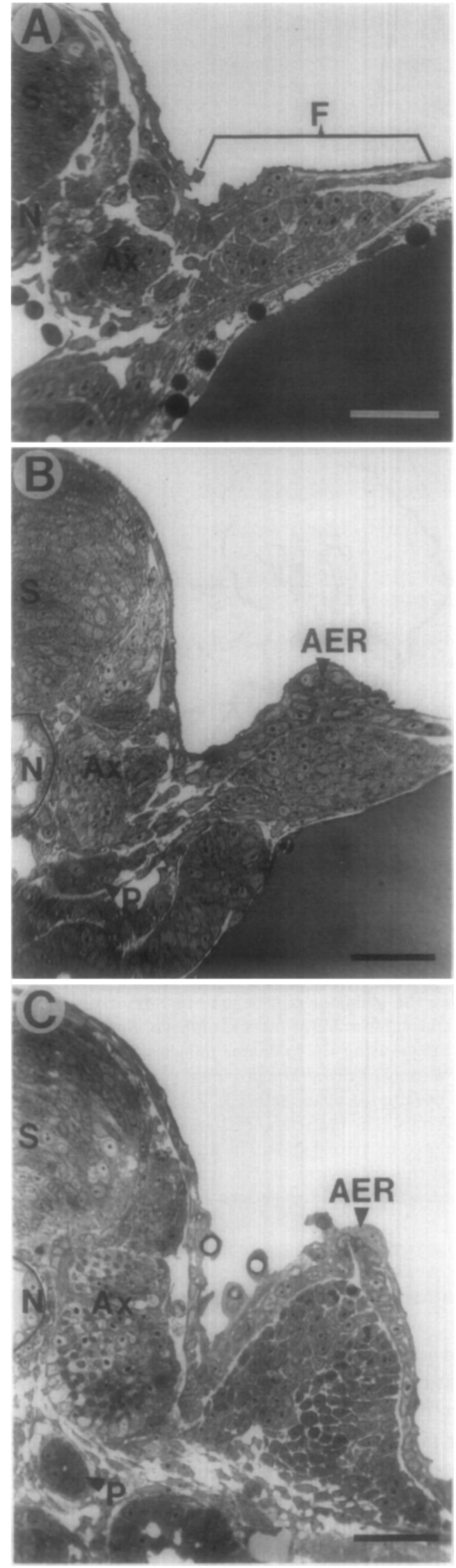

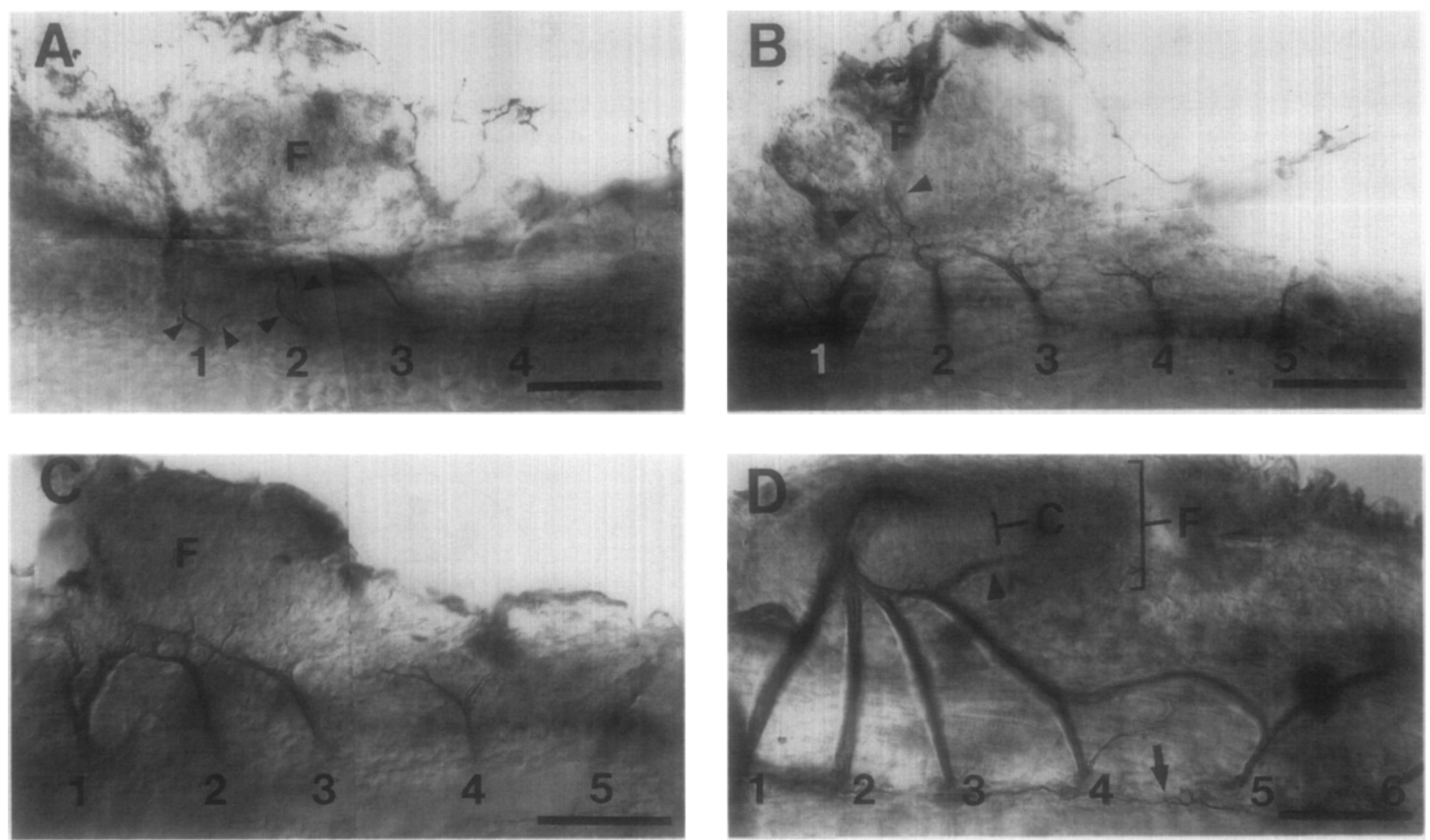

Fig. 4. Pattern of nerve outgrowth from S1-5. Ventral views of embryos with segmental nerves labeled with the acetylated tubulin antibody. Anterior is to the left and lateral is up. (A) Photumontage of a 60-hr embryo showing that S1-3 nerves are directed towards the base of the fin bud, F, early during outgrowth and, in some cases, branch (arrowheads) but the S4-5 nerves do not. In this case there were four branches of the S1 nerve, four branches of the S2 nerve, and the S3 nerve was unbranched. Some of the branches cannot be seen in this photograph since they were at different focal planes. Anterior, left; lateral, up. (B) Photomontage of a 64-hr embryo showing the S1-3 nerves are converging. In this case there were seven branches of the S1 nerve, five branches of the S2 nerve, and six branches of the S3 nerve. Again some of the branches were at different focal planes. Some branches (arrowheads) can be seen entering the fin bud prior to convergence of the segmental nerves. The S4 nerve is branching laterally with branches directed anteriorly and posteriorly. (C) Photomontage of a 70-hr embryo showing that the S1-3 nerves have converged to form a plexus and that branches have entered the fin bud. (D) A 121-hr embryo with nerves from S1-5 converged and adductor and abductor muscle nerves emerging from the plexus. The branch from $\mathrm{S} 4$ which turns away from the fin bud is denoted by an arrowhead. S1 also has a branch turning away from the fin bud but it is out of the focal plane. The cilia (arrow) in the lumen of the pronephros are also labeled by the acetylated tubulin antibody and can be seen in the bottom of the micrograph. C, cartilage; scale for (A-D), $50 \mu \mathrm{m}$.

4C). Most of the branching seen at this stage occurs at the level of or beyond the plexus. The $\mathbf{S 4}$ nerve now has a branch directed anteriorly towards the fin bud and a branch running posteriorly. The main trunk of the $\mathrm{S} 1$ nerve has branched with one branch entering the plexus and the other branch extending in an anterior direction. By $84 \mathrm{hr}$ (stage 29) when the mesenchyme has coalesced into the two muscle blocks separated by nascent cartilage, two thick nerves on either side of the nascent cartilage and corresponding to the two muscle nerves have extended from the plexus ( $n=3$; data not shown). Later at $121 \mathrm{hr}$ (stage $31 ; n=4$ ) numerous branches have extended perpendicular from the two muscle branches into the nascent adductor and abductor muscle masses (not shown), the thick branch of the S4 nerve has converged with the $\mathrm{S} 3$ nerve, and a posteriorly directed branch has emerged from the S4 nerve near the plexus (Fig. 4D). Additionally, the anterior branch of the S1 nerve now has a ventral trajectory (data not shown).
Subsequently by 10 days the posterior branch of $\mathrm{S} 4 \mathrm{ex}-$ tends in a posterior/ventral direction (see above).

Cross sections of embryos with labeled axons showed that the S2 3 axons initially extend between the ventral surface of the axial muscles and the pronephros and then, once past the pronephros, on the muscle (not shown) to reach the fin bud. Light microscopic examination indicates no obvious structures such as blood vessels, grooves, or septa which demarcated the pathway taken by the axons. Furthermore, the pathway runs oblique to the longitudinal orientation of the axial muscles.

Outgrowth by segmental nerves from segments posterior to S4 differs from that found in S1-4. Axons from S5 reach the ventral surface of the axial muscles in their segments by $64 \mathrm{hr}$ and extend laterally and posteriorly (Figs. $4 \mathrm{~B}$ and $4 \mathrm{C}$ ). The nerves posterior to S5 usually do not branch at this time and they extend along a pathway that is parallel to the borders between axial muscle seg- 

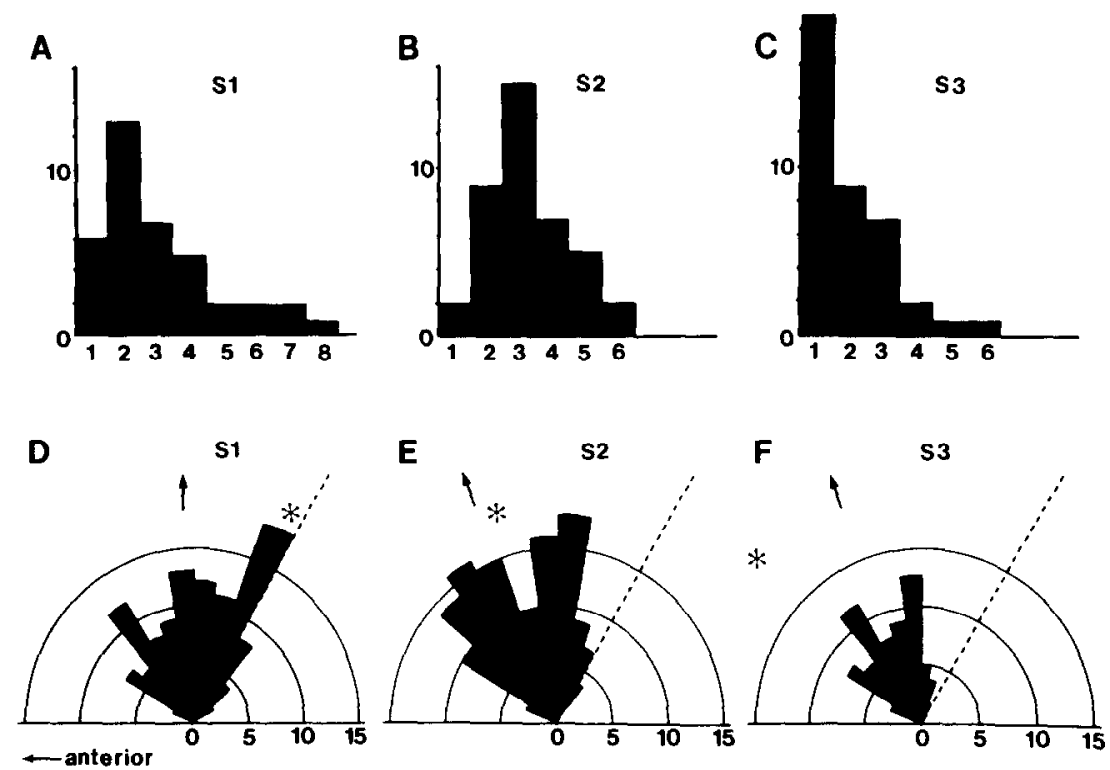

Fig. 5. Branching by nerves from S1-3 and directionality of these branches in 58-64-hr embryos. Data was quantified from embryos with nerves labeled with the acetylated tubulin antibody. (A-C) Incidence of branching by the S1 nerve (A); the S2 nerve (B); and the S3 nerve (C). Ordinate, number of cases; abscissa, number of branches per nerve. (D-F) Directionality of nerve branches for the S1 nerve, (D); Sz nerve, (E); and S3 nerve, (F). Polar plots of the distribution of the tips of nerve branches on the ventral surface of the axial muscle. Anterior is to the left. See Materials and Methods for details. Dotted line represents the direction of the segment border. Each sector represents $10^{\circ}$. Arrows indicate the average direction of the nerve branches and asterisk the direction of the center of the limb bud.

ments ( $n=8$; data not shown). Near the lateral edge of the muscles these nerves turn posteriorly and taper down but appear to remain in their respective segments (data not shown; see also Fig. 8 F). In seven of 8 cases an anteriorly directed branch connected the S5 nerve with the $\mathrm{S} 4$ nerve near the lateral edge of the axial muscles by $121 \mathrm{hr}$ (stage 31; Fig. 4D). This connection may be eliminated later in development since by Day 10 only 1 of 7 S5 nerves had such a connection (sec above).

\section{Pathfinding by Motor Growth Cones}

The relationship of motor growth cones and their filopodia to their environment was determined by orthogradely labeling them by injections of diI to the ventral cord (Figs. 6A-D). At 52-56 hr (stage 24 to early stage 26) growth cones from S1-5 extend ventrally between the notochord and the medial surface of the axial muscles $(n=6)$. The growth cones from S1-3 tend to extend straight ventrally while the S5 growth cone follows a pathway which is parallel to the borders of the axial muscles and, therefore, is deviated in a slightly posterior direction. A difference between the S1-3 nerves and the S4-5 nerves along this portion of their trajectories is especially clear in later embryos in which the segmental nerves have been labeled with the antibody against ace- tylated tubulin (Fig. 6E). Since the axial motor axons share much of the pathway taken by fin motor axons it was not possible to identify whether these growth cones were those of axial or fin motor axons or both.

By 56 to $64 \mathrm{hr}$ (stage 26 to early stage $27 ; n=6$ ) growth cones from S1-2 have reached and begun to extend on the ventral surface of the axial muscles. The S1 growth cones and their filopodia were directed both anteriorly and posteriorly in concordance with the pattern of nerve branching seen in acetylated tubulin-labeled embryos (data not shown). S2 growth cones and their filopodia are directed towards the base of the fin bud (Figs. 6C and $6 \mathrm{D}$ ). These growth cones are large and a group of growth cones and their filopodia can span up to $25 \mu \mathrm{m}$ from side to side; and therefore, theoretically they could sample a large proportion of the ventral surface of the axial muscles in one segment, which typically measures 40 by $50 \mu \mathrm{m}$. In concordance with the branching seen with the acetylated tubulin antibody, growth cones were predominantly directed towards the fin bud but were often dispersed. In these preparations we often found one or a small group of growth cones which preceded the others (Figs. 6C and 6D).

In sections of embryos with fin nerves labeled with the acetylated tubulin antibody we saw no obvious cells or structures which demarcate the pathways taken by the fin axons (see above). This was confirmed by light 

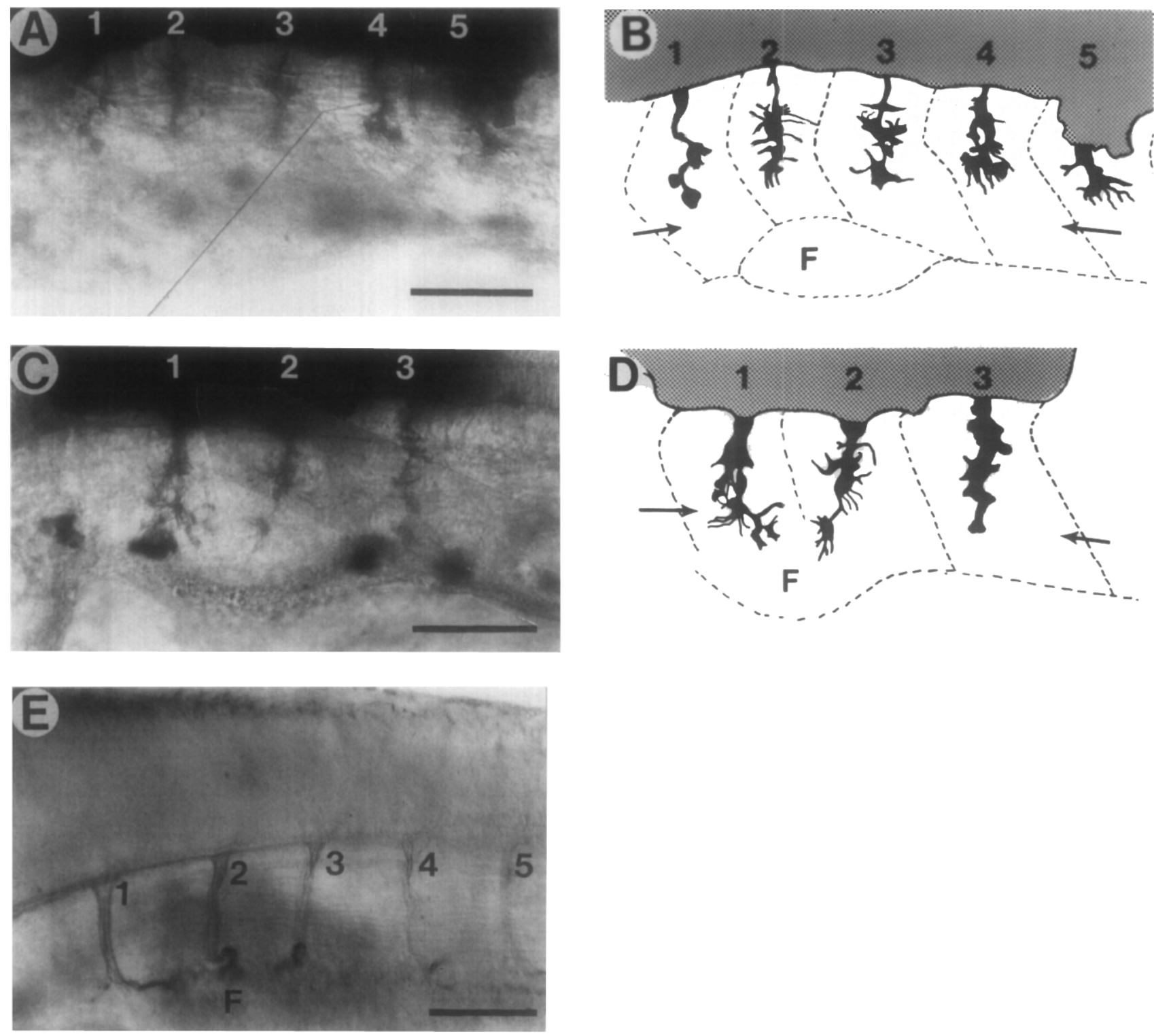

FIG. 6. Extension of growth cones from motor neurons. Motor growth cones were labeled orthogradely by injection of diI into the ventral spinal cord followed by photooxidation (A-D) or nerves were labeled with the acetylated tubulin antibody (E). (A) Photomontage of a lateral view of growth cones extending ventrally in a 54-hr embryo. (B) Camera lucida drawing of growth cones shown in (A). The medial border of the ventral surface of the axial muscles is denoted by the arrows. (C) Photomontage of a lateral view of growth cones extending in a 57 -hr embryo. The S1 and S2 growth cones have reached the ventral surface of the axial muscles and begun to extend laterally towards the nascent fin bud $\mathrm{F}$. (D) Camera lucida drawing of the growth cones shown in (C). Scale for A-D, $50 \mu \mathrm{m}$. (E) The ventrally running portion of nerves from $\mathrm{S} 1-3$ differ in trajectory from that of nerves from S4-5. Lateral view of a 70-hr embryo. The proximal portion of nerves from S1-3 run straight ventrally while that of nerves from S4-5 run ventrocaudally. Scale, $50 \mu \mathrm{m}$.

microscopic analysis of sections of fin growth cones labeled with diI and then marked with the DAB reaction product following photooxidation $(n=4)$. Sections distal to, through, and behind the growth cones showed no patterned set of cells (living or dying) or structures that may potentially act as a preformed pathway on the ventral surface of the axial muscles as could be ascertained by these methods (Fig. 7).
Stereotyped Pattern of Segmental Nerves from $S_{1-6}$ in Late Embryos

We described the pattern of the segmental nerves innervating the fin bud by labeling axons with the acetylated tubulin antibody (see above). However, once axons merge with each other in the plexus it was impossible to determine their trajectories. Furthermore, thin 

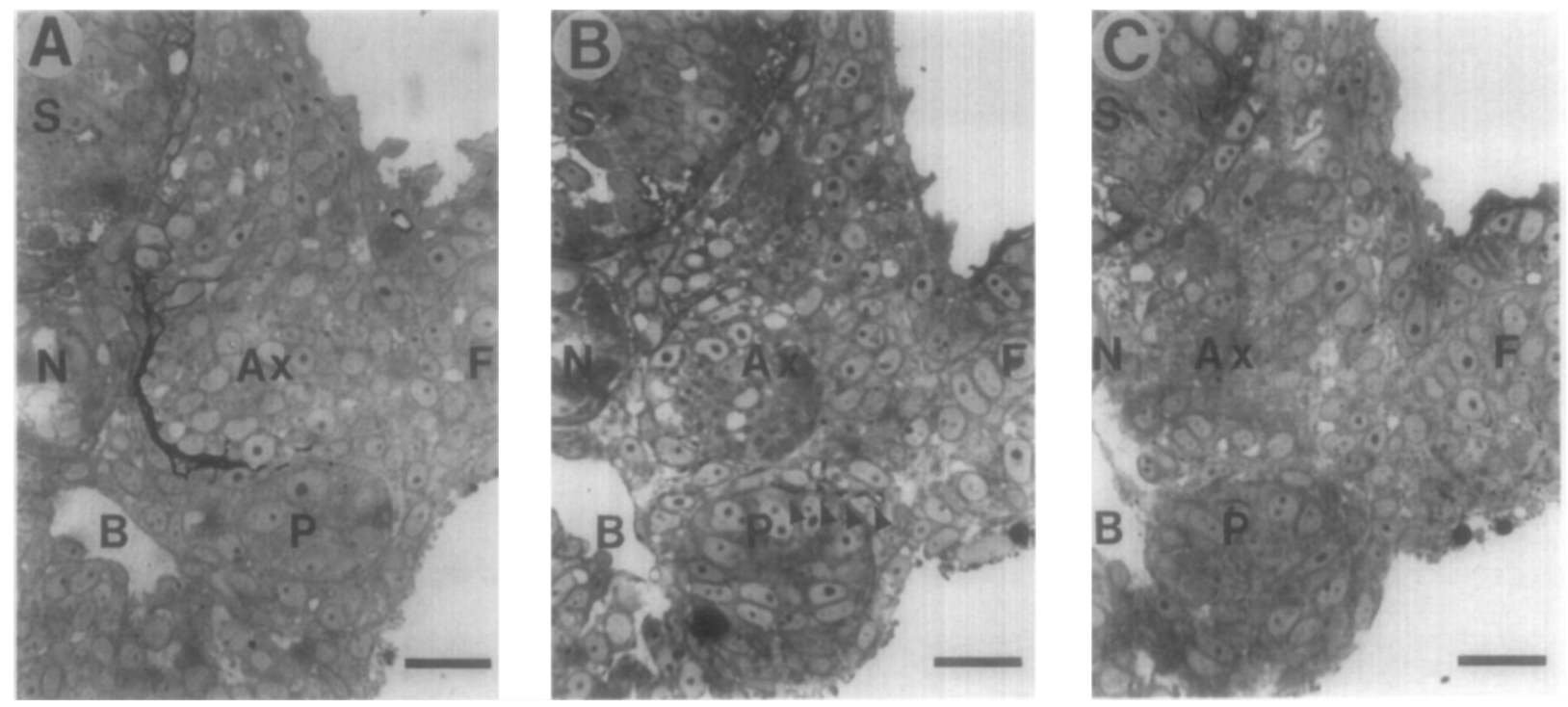

Fig. 7. No obvious structures demarcate the pathways taken by growth cones extending towards the fin bud. Cross sections of the 57-hr embryo with dil-labeled grow th cones shown in Fig. 6C and 6D. (A) Section showing labeled axons in the S2 nerve approximately $5 \mu \mathrm{m}$ posterior to the most distal growth cones. S, spinal cord; N, notochord; Ax, axial muscles; B, blood vessel; P, pronephros; F, fin bud. (B) Section at the level of the most distal growth cones and filopodia (arrowheads). (C) Section approximately $5 \mu \mathrm{m}$ anterior to the most distal S2 growth cones. Scale for (A-C), $10 \mu \mathrm{m}$.

branches which may invade the axial muscles from their ventral surface may not be accessible to the acetylated tubulin antibody. Therefore, the pattern of axons from each segment (S1-6) was determined by labeling them by application of diI to the ventral spinal cord or the ventral roots in $84 \mathrm{hr}$ (stage 29 ) embryos ( $n=14$; Fig. 8). Each nerve had a stereotyped trajectory and branching pattern.

As described before, the S1 nerve branched prior to the plexus with one branch entering the plexus and the other turning anteriorly. In accord with the back-fill data from the two muscles, $\mathrm{S} 1$ axons which enter the plexus extend into both the adductor and abductor muscle nerves. The main trunk of the S1 nerve did not project any branches along the ventral surface of the axial muscles. Likewise, the $\mathrm{S} 2$ axons emerged from the plexus in two bundles, one for each muscle, and had no axial muscle branches. However, the S3 nerves projected many side branches from the main trunk on the ventral surface of the axial muscles. These branches ran posteriorly and parallel to the axial muscle fibers and may represent axons of motor neurons which innervate the axial muscles from the ventral surface of the axial muscles. However, we cannot rule out that these axons may be collateral branches of the fin motor axons. S3 axons like the $\mathrm{S} 1$ and $\mathrm{S} 2$ axons emerged from the plexus in two bundles to innervate both fin muscles. S4 has a major branch which runs anteriorly and a minor branch posteriorly. There are many side branches from the main trunk and posterior branch of S4. S5 possesses a minor branch which runs anteriorly and a major branch which remains parallel to the muscle segment borders. S6 has only a main trunk running in parallel with the segment borders. The main trunk of the nerves from S5-6 have numerous side branches similar to those seen in S3.

\section{Development of the Fin Bud in pl Embryos}

The $p l$ mutation is a spontaneously generated, viable, and recessive mutation that leads to adult Japanese medaka fish which are missing pectoral fins (Tomita, 1982). Despite this the pectoral fin bud does undergo the earliest stages of development (up to $70 \mathrm{hr}$ approximately) before arresting in $p l$ embryos (Fig. 9). Just as in wildtype embryos, the first morphological signs of the pectoral fin bud is a slight swelling on the yolk sack centered at the border between S1 and S2 at $60 \mathrm{hr}$ (late stage $26 ; n=8$ ). Ten hours later the fin bud has increased in size and the ectoderm overlying the distal tip begins to differentiate into the AER $(n=10)$. By $84 \mathrm{hr}$ (stage 29) the fin bud mesenchyme has coalesced into three blocks of tissue, the two nascent muscles separated by nascent cartilage, in wildtype embryos; but in $p l$ embryos the mesenchyme fails to differentiate and the fin bud has become very flat $(n=4)$.

\section{Outgrowth of Fin Motor Nerves in pl Embryos}

We assessed outgrowth of fin motor axons in $p l$ embryos by labeling segmental nerves with the acetylated 

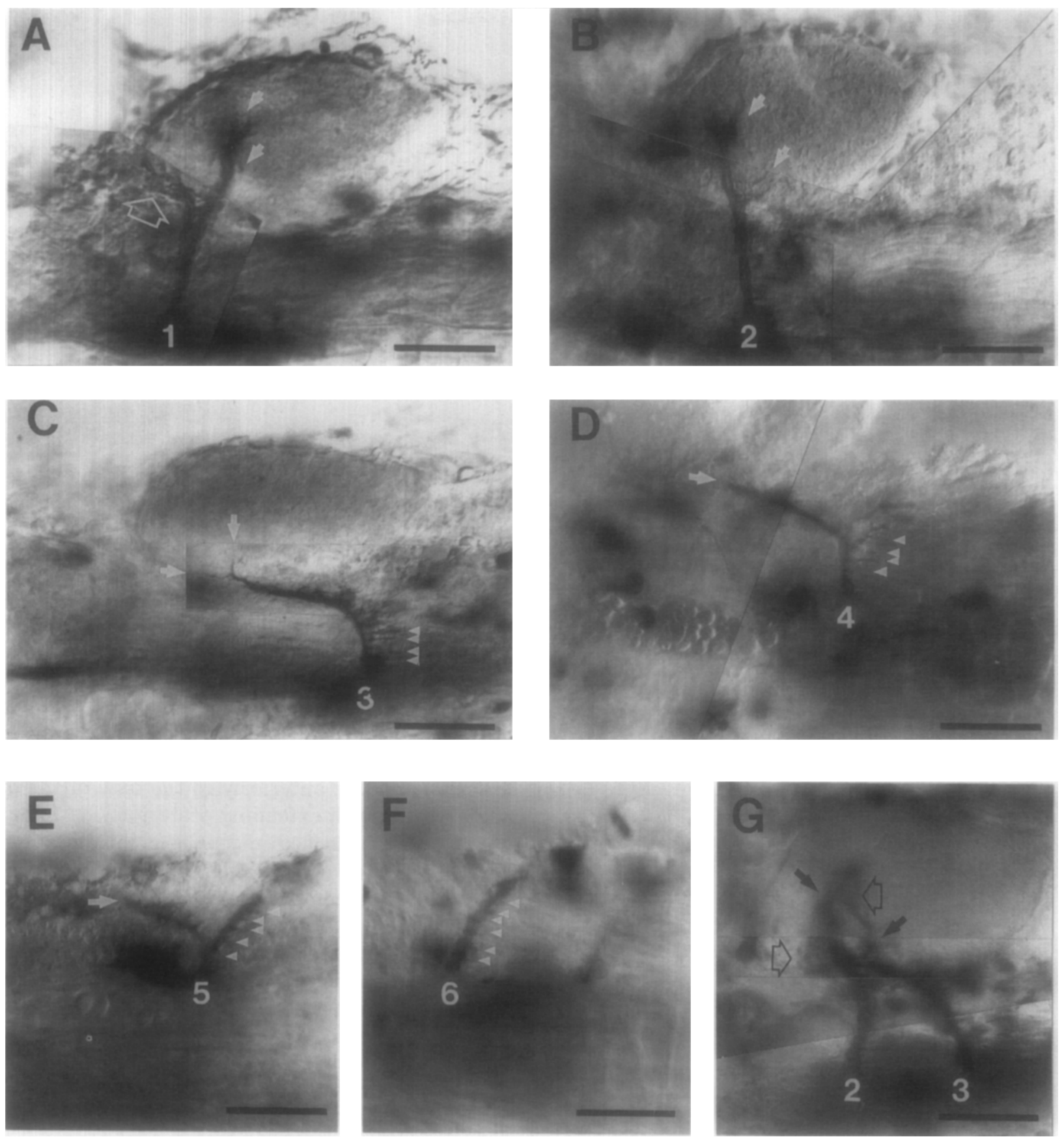

FIG. 8. Stereotyped pattern of nerves from S1-6 in embryos approximately 84 hr old. Nerves were orthogradely labeled with diI followed by photooxidation. All micrographs are ventral views. Anterior is to the left and lateral is up. (A) S1 nerve. Open arrow, branch of S1 running anteriorly away from the plexus; upper closed arrow, the adductor muscle branches which are out of focus; lower closed arrow, small abductor muscle branch. (B) S2 nerve. Upper and lower arrows denote the adductor and abductor musele branches, respectively. (C) S3 nerve. Upper and lower arrows denote the nascent adductor and abductor muscle branches. Arrowheads indicate side branches running posteriorly along the axial muscle fibers. (D) The S4 nerve has not yet entered the fin bud. Arrow, distal tip of the anterior branch of the S4 nerve: arrowheads, same as in (C). (E) The $\mathrm{S} 5$ nerve has branched but the anterior branch has not reached the fin bud while the posterior branch has numerous side branches (arrowheads). (F) S6 nerve with numerous side branches (arrowheads). (G) S2 and S3 nerves. Closed arrows, adductor (upper) and abductor (lower) branches of the S2 nerve; open arrows, adductor (upper) and abductor (lower) branches of the S3 nerve. Scale for (A-G), b0 $\mu \mathrm{m}$.

tubulin antibody. As in wildtype embryos nerves from S1-4 had reached and begun to extend on the ventral surface of the axial muscles by $60 \mathrm{hr}(n=7$; compare Figs. 4A and 10A). The nerves from segments posterior to S5 were indistinguishable from those of wildtype embryos and the nerves from S1-3 did converge at the base of the fin bud by $70 \mathrm{hr}$, but the pattern of outgrowth differed from wildtype in four ways $(n=40$; compare 

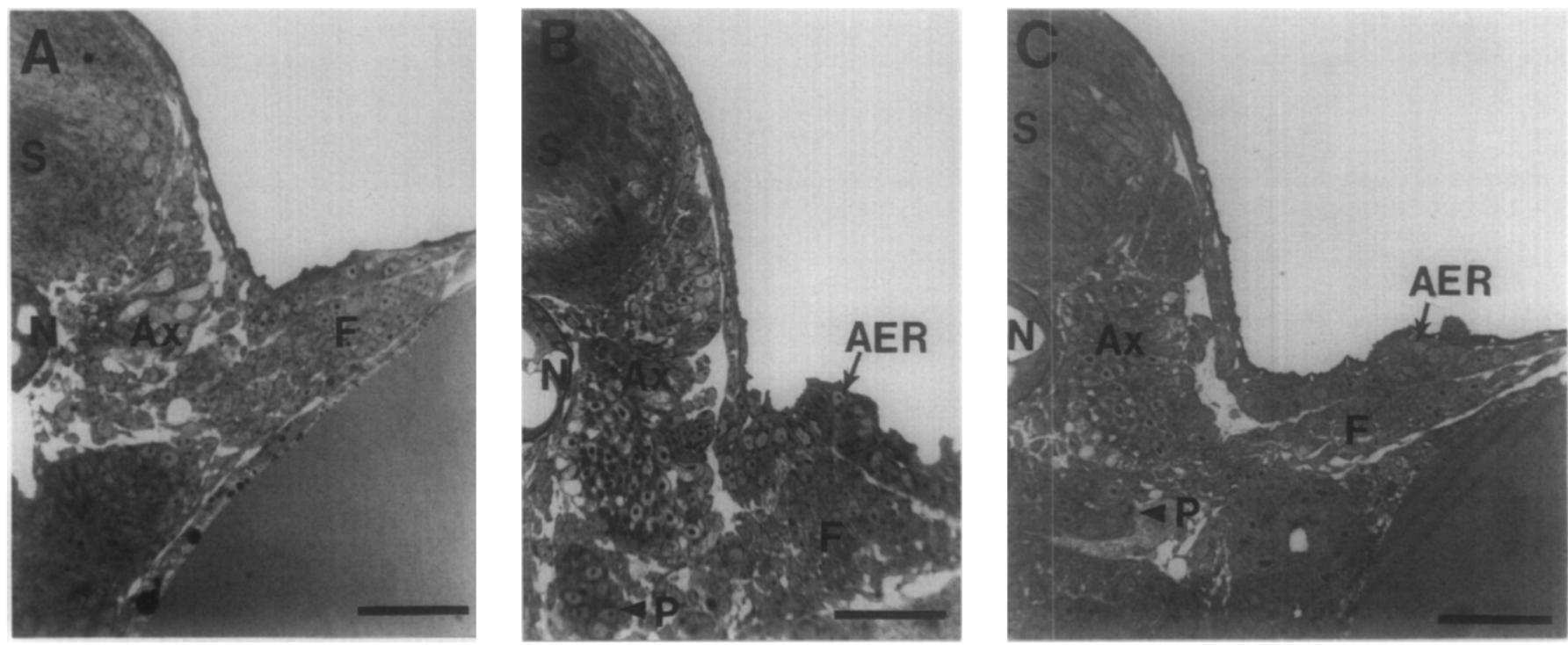

Fig. 9. The arrest of fin buds in $p l$ embryos. Cross sections of plastic-embedded embryos at $60 \mathrm{hr}(\mathrm{A}), 70 \mathrm{hr}$ (B), and $84 \mathrm{hr}$ (C) of development. S, spinal cord; N, notochord; Ax, axial muscles; P, pronephros; F, fin bud; AER, apical ectodermal ridge. Scale for (A-C), $20 \mu \mathrm{m}$.

Figs. 4C and 10B). (1) The S3 nerve initially ran laterally along a trajectory which was parallel to the anterior and posterior borders of the ventral surface of the axial muscles much like the trajectory of the segmental
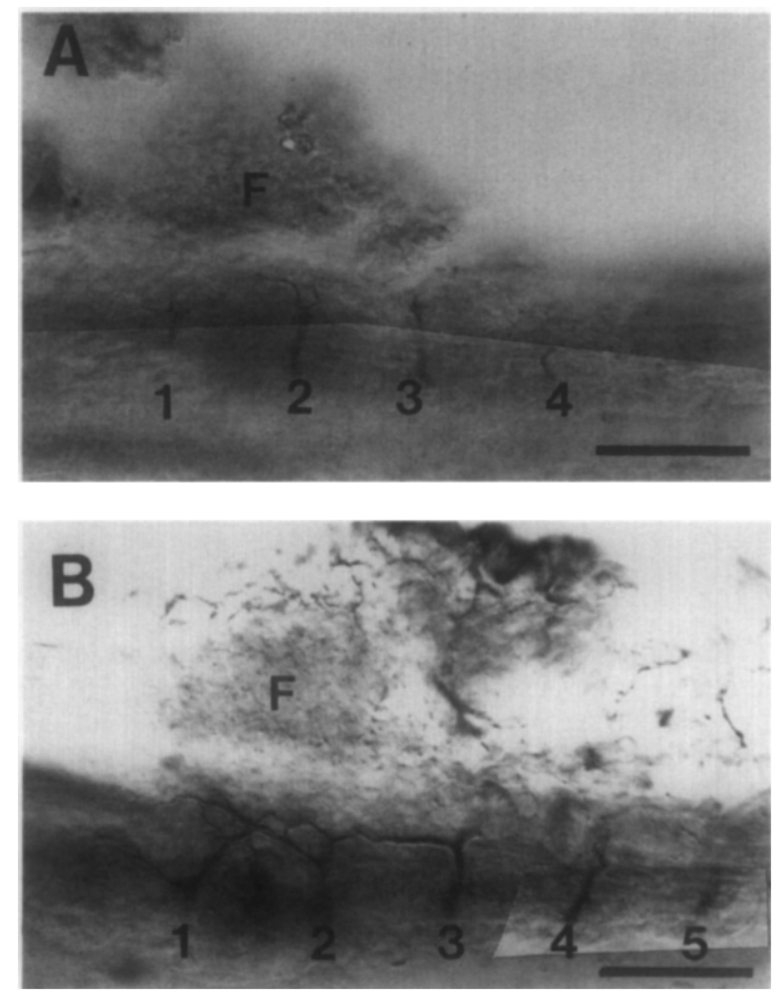

FIG. 10. Outgrowth by nerves from $\$ 1-5$ in $p l$ embryos. All panels are ventral views of acetylated tubulin labeled embryos. Anterior, left; lateral, up. (A) pl embryo at $60 \mathrm{hr}$ of development. (B) pl embryo at $70 \mathrm{hr}$ of development. Seale for (A, B), $50 \mu \mathrm{m}$. nerves posterior to $\mathrm{S} 4$ rather than diagonally towards the base of the fin bud. The S3 nerve did eventually converge with the $\mathrm{S} 1$ and $\mathrm{S} 2$ nerves to form a plexus at the base of the fin bud, but did so by making a sharp anterior turn on the lateral portion of the axial muscles in $p l$ embryos. (2) Normally the S4 nerve branched on the ventral surface of the axial muscles with a thick branch extending anteriorly to the base of the fin bud and a thin branch extending posteriorly by $70 \mathrm{hr}$. In pl the $\mathrm{S} 4$ nerve branched in four of eight cases at $70 \mathrm{hr}$. When the S4 nerve did branch, it did so in a much more lateral position compared to the $\mathbf{S} 4$ nerve in wildtype embryos and the anterior branch was much thinner and shorter than normal. In three of eight cases the S4 nerve had a thin anterior branch that ran into the plexus in 84-102-hr $p l$ embryos. In these cases the $\mathrm{S} 4$ nerve resembled the nerve from S5 in wildtype embryos. In the other five cases, the $\mathrm{S} 4$ nerve did not branch but instead ran laterally and posteriorly as normally the nerves in more posterior segments do (not shown). (3) The S5 nerve in 84-hr $p l$ embryos $(n=8)$ had not branched as was usual in wildtype embryos, and as a consequence resembled nerves from more posterior segments (not shown). (4) Normally axons extend from the plexus to innervate the two fin muscles by $84 \mathrm{hr}$, but in $p l$ axons these have not grown into the fin bud by this time ( $n$ $=4)$.

\section{DISCUSSION}

\section{Pattern of Outgrowth by Limb Motor Axons}

The pattern of limb nerves is stereotyped in amphibians and chicks as well as in fish embryos. The growth 
cones of fin motor neurons reach the base of the fin bud by extending in a directed fashion toward it. Previously, brachial nerves were traced in chicks and observed to grow out to the base of the wing bud in a similar fashion (Bennett et al., 1980). Our study has extended this finding by observing pathfinding by motor growth cones to the base of the fin bud. The directed extension of growth cones towards the base of the fin bud is evident both on the medial and the ventral surfaces of the axial muscles. On the latter the growth cones and filopodia and the trailing axons are directed and extend towards the base of the fin bud.

What might account for convergence by the growth cones from S1-4? Although the growth cones and filopodia can cover a significant amount of territory as judged by diI fills, they are still too small to contact the fin bud directly upon emergence onto the ventral surface of the axial muscles. This makes it unlikely that contact of the fin bud by randomly projected filopodia from these growth cones can exclusively account for pathfinding by these growth cones. This is reinforced by the finding that motor axons will extend to a donor fin bud located lateral to the host fin bud and, therefore, clearly beyond the range of their filopodia (Okamoto and Kuwada, 1991). Additionally, on the portion of the pathway associated with the ventral surface of the segmented axial muscles there are no obvious cells or structures which may act as a local guidance cue or a preformed pathway. There are no blood vessels, appropriately patterned set of mesenchyme cells or dying cells, septa, cartilagenous structures, grooves, or holes which may be influencing the pattern of outgrowth. Furthermore, the pathways followed by the growth cones ignore the orientation of the axial muscles. The lack of any cells or structures which may operate as local guidance cues or preformed pathways does not preclude any involvement of local cues and preformed pathways, which may merely be undetectable with the methods used in this study, for pathfinding by the motor growth cones. In fact in the chick some structures, such as the sclerotome, nascent pelvic girdle, and perinotochordal mesenchyme do in part influence the pattern of outgrowth by hindlimb motor axons (Keynes and Sterns, 1984; Tosney and Landmesser, 1984, 1985; Tosney and Oakley, 1990).

The behavior of the earliest motor growth cones and axons during convergence appears to be more consistent with the hypothesis that the fin bud attracts motor growth cones, perhaps via a long distance cue, than a spatially restricted set of local cues. First, the motor growth cones are large and cover a significant proportion of their substrate. Second, the earliest axons usually diverge from each other but are, nevertheless, predominantly directed towards the fin bud. Third, the trajectories of the segmental nerves posterior to the fin bud change systematically with distance from the fin bud. The S5 nerve is the farthest of the fin nerves and is the one most like the nerves from segments which do not innervate the fin. It only extends a thin, possibly transient branch to the fin bud. The $\mathrm{S} 4$ nerve is next farthest and extends a major branch to the fin bud while the entire nerve from $\mathrm{S} 2$ and $\mathrm{S} 3$ converge towards the fin bud. One might expect these observations if the growth cones were actually sampling a long distance cue emanating from the fin bud.

\section{Nerve Outgrowth in Limb Mutants}

The pattern of outgrowth by the S1-5 nerves in $p l$ mutants is consistent with the hypothesis that the fin bud normally attracts the S1-5 nerves to the base of the fin bud. It is possible that the $p l$ fin bud has a diminished capacity to attract segmental nerves compared to the wildtype fin bud. Three abnormalities in the pattern of outgrowth in $p l$ correspond to a diminished capacity on the part of the fin bud, motor axons, or both. (1) The S5 nerve, which is farthest from the fin bud of the nerves which normally send axons to the plexus, does not project any axons to the fin bud much like nerves from segments posterior to S5. (2) The S4 nerve, which is the next farthest from the fin bud, only converges about half the time. When it does converge, it resembles the S5 nerve in wildtype embryos: it branches near the lateral edge of the axial muscles and the anterior branch is thin. In the other cases it has a trajectory similar to that of nerves posterior to S5. (3) The pattern of outgrowth by the S3 nerve, which is closer to the fin bud than $\mathrm{S} 4$ but farther than $\mathrm{S} 1$ or $\mathrm{S} 2$, may represent another intermediate effect. The S3 nerve initially ran parallel to the anterior and posterior borders of the axial muscle to the lateral portion of the muscle much like nerves from segments posterior to $\mathrm{S} 4$ before abruptly turning anterior towards the fin bud. The more proximal portion of the S3 trajectory resembles that of the nerves from more posterior segments. The attraction signal may be more readily accessible laterally than medially. Any diminished capacity of the fin bud or motor growth cones in $p l$ probably cannot be accounted for by any obvious morphological defect: much of the convergence by fin nerves has occurred by $70 \mathrm{hr}$ in wildtype embryos, and in $p l$ embryos the morphological development of the fin bud appears normal to $70 \mathrm{hr}$ but the pattern of convergence is already abnormal. Outgrowth by motor axons in other limb mutations in various species may illuminate possible structures in the fin bud which may be necessary for convergence by the motor axons to the base of the limb bud. For example, in limbless mutants in chicks it is possible that the development of the limb bud is arrested even earlier than in $p l$ since the AER never forms in 
limbless (Prahlad et al., 1979). It is known that motor neurons in limbless can be rescued from cell death by a transplanted wildtype limb bud signifying that some aspects of these motor neurons are normal (Lanser et al., 1986); and at least some segmental nerves get to the limbless limb bud (Lanser and Fallon, 1984), but whether all the limb nerves converge or how their pathways may be affected by the mutation is unknown.

\section{Attraction of Axons by Limb Buds}

Transplantation of limb buds in amphibian and chick embryos has also suggested that axons are attracted by limb buds (Hamburger, 1939; Detwiler, 1934, 1936). More recently in vitro experiments have demonstrated that axons can be attracted by long distance cues in the mammalian spinal cord, brain, and peripheral nervous system (Lumsden and Davies, 1983, 1986; Heffner et al., 1990; Tessier-Lavigne et al., 1988).

If convergence by the motor axons is due to attraction of growth cones by the fin bud, the fin bud may do so in a nonspecific manner, as do the limb buds in amphibians and chicks (Detwiler, 1934, 1936; Hamburger, 1939). This may be the case since nonfin axons also extend towards the fin. (1) In the main trunk of the S1 and S4 nerves are axons of an unidentified population of neurons which initially extend towards the base of the fin bud but then turn away. (2) In the S3 and S4 nerves are axons which may represent the axons of axial motor neurons which innervate the ventral-most axial muscles. These axons also extend partially towards the fin bud before branching along the axial muscles. Alternatively, the nonfin axons in the segmental nerves may follow the fin axons before following another set of cues rather than be directly attracted by the fin bud.

The role the fin bud plays for convergence by motor axons can be assessed by manipulations of the fin. Successful mechanical manipulation of the fin bud is greatly enhanced by several geometric factors. First, the fin bud is located in S2 while some of the motor neurons innervating it are located far enough away so that their pathways are not disturbed following, for example, ablation of the fin bud. Second, the fin bud is located lateral to the main body and oriented such that the fin bud can be completely removed without damaging tissues and structures which are often contiguous with the base of the limb bud in other systems. The companion paper (Okamoto and Kuwada, 1991) describes motor nerve outgrowth following both ablation and transplantation of the fin bud.

We thank H. Tomita, A. Shima, and K. Naruse for fish stocks; G. Piperno, for the acetylated tubulin antibody; P. Knafl for technical assistance; D. Bay for photography; and H. Tomita, R. R. Bernhardt, and $\mathrm{K} . \mathrm{W}$. Tosney for reading the manuscript. This work was supported by National Institute of Health Grant NS24848 and March of
Dimes Grant 5-687. J.Y.K. is a Basil O'Connor Scholar of the March of Dimes and H.O. is a postdoctoral fellow of the Toyobo Biotechnology Foundation.

\section{REFERENCES}

ABERCRombie, M. (1946). Estimation of nuclear population from microtome section. Anat. Rec. 94, 239-247.

BenNeTt, M. R., DAveY, D. F., and Uebel, K. E. (1980). The growth of segmental nerves from the brachial myotomes into the proximal muscles of the chick forelimb during development. J. Comp. Neurol. 189, 335-357.

Bernhardt, R. R., Chitnis, A. R., Lindamfr, L., and Kuwada, J. Y. (1990). Identification of spinal neurons in the embryonic and larval zebrafish. J. Comp. Neurol. 302, 603-616.

BOUVET, J. (1978). Cell proliferation and morphogenesis of the apical ectodermal ridge in the pectoral fin bud of the trout embryo (Salmo trutta fario. L.) Wilhelm Roux Archiv. 185, 137-154.

ChitNis, A. B., and KuwadA, J. Y. (1990). Axonogenesis in the brain of zebrafish embryos. $J$, Neurosci. 10, 1892-1905.

DETwiLER, S. R. (1934). An experimental study of spinal nerve segmentation in Amblystoma with references to the plurisegmental contribution to the brachial plexus. J. Exp. Zool. 67, 395-441.

Detwller, S. R. (1936). "Neuroembryology," Haffner, New York.

HAMBURGER, V. (1929). Experimentelle Beiträge zur Entwicklungsphysiologie der Nervenbahnen in der Froshextremität. Wilhelm Roux Arch. 119, 47-99.

HAMBURGER, V. (1939). The development and innervation of transplanted limb primordia of chick embryos. J. Exp. Zool. 80, 147-189.

Hanker, J. S., Yates, P. E., Metz, C. B., and Rustoni, A. (1977). A new specific sensitive and nonearcinogenic reagent for the demonstration of horse-radish peroxidase. Histochem. J. 9, 789-792.

HeFFNER, D. C., Lumsden, A. G. S., and O'Leary, D. D. M. (1990). Target control of collateral extension and directional axon growth in the mammalian brain. Science 247, 217-220.

HoNIG, M. G., and HumE, R. I. (1986). Fluorescent carbocyanin dyes allow living neurons of identified origin to be studied in long-term cultures. J. Cell Biol. 103, 171-187.

KEynES, R. J., and STERN, C. D. (1984). Segmentation in the vertebrate nervous system. Nature 310, 786-789.

KIRCHEN, R. V., and WEST, W. R. (1976). "The Japanese Medaka, Its Care and Development." Carolina Biological Supply Co. Burlington. NC.

KuWADA, J. Y. (1986). Cell recognition by neural growth cones in a simple vertebrate embryo. Science 253, 740-746.

KuWAdA, J. Y., BERNHARDT, R. R., and NguYEN, N. (1990). Development of spinal neurons and tracts in the zebrafish embryo. J. Comp. Neurol. 302, 617-628.

LANDMESSER, L. (1988). Peripheral guidance cues and formation of specific motor projections in the chick. In "From Message to Mind" (S. Easter, K. Barald, and B. Carlson, Eds.), pp. 121-133. Sinauer, Sunderlind, MA.

LANSER, M. E., and Fallon, J. F. (1984). Development of the lateral motor column in the limbless mutant chick embryo. J. Neurosci. 4, 2043-2050.

LanSer, M. E., Carrington, J. L., and Fallon, J. F. (1986). Survival of motorneurons in the brachial lateral motor column of limbless mutant chick embryos depends on the periphery. J. Neurosci. 6, 25512557.

Lumsden, A. G. S., and DAvies, A. M. (1983). Earliest sensory nerve fibres are guided to peripheral targets by attractants other than nerve growth factor. Nature 306, 786-788.

Lumsden, A. G. S., and Davies, A. M. (1986). Chemotropic effect of specific target epithelium in the developing mammalian nervous system. Nature 323, 538-539. 
MaRANTO, A. R. (1982). Neuronal mapping: A photooxidation reaction makes Lucifer Yellow useful for electron microscopy. Science 217, $953-955$.

OKaмото, H., and KuWADA, J. Y. (1991). Alteration of pectoral fin nerves following ablation of fin buds and by ectopic fin buds in the Japanese Medaka fish. Dev. Biol. 146,

Patel, N. H., Martin-Blanco, E., Coleman, K. G., Poole. S. J., Ellis, M. C., Kornberg, T. B., and Goomman, C. S. (1989). Expression of engrailed protein in arthropods, annelids, and chordates. Cell 58, 955-968.

Piperno, G., and Fuller, M. T. (1985). Monoclonal antibodies specific for an acetylated form of $\alpha$-tublin recognize the antigen in cilia and flagella from a variety of organisms. $\int$. Cell Biol. 101, 2085-2094.

Prahlad, K. V., Skala, G., Jones, D. G., and Briles, W. E. (1979). Limbless: A new genetic mutant in the chick. $J$. Exp. Zool. 209, 427434.

SPERRY, R. W. (1950). Myotypic specificity in teleost motorneurons. $J$. Comp. Neurol 93, 277-288.

Tessier-Lavigne, M., Placzek, M., Lumsden, A. G. S., Dodd, J., and Jessel, T. M. (1988). Chemotropic guidance of developing axons in the mammalian central nervous system. Nature $336,775-778$.
Tomita, H. (1982). Gene analysis in the Medaka (Oryzias latipes). Medaka 1, 7-9.

TOSNEy, K. W., and Landmesser, L. T. (1984). Pattern and specificity of axonal outgrowth following varying degrees of chick limb bud ablation. J. Neurosci. 4, 2158-2527.

Tosney, K. W., and Landmesser, L. T. (1985). Development of the major pathways for neurite outgrowth in the chick hind limb. Dev: Biol. 109, 193-214.

Tosney, K. W., Schroeter, S., and Pokrzywinski, J. A. (1988). Cell death delineates axon pathways in the hind limb and does so independently of neurite outgrowth. Dev. Biol. 130, 558-572.

TOSNEY, K. W., and OAKLEy, R. A. (1990). The perinotochordal mesenchyme acts as a barrier to axon advance in the chick embryo: Implications for a general mechanism of axonal guidance. Exp. Neurol. $109,95-89$.

Wood, A. (1982). Early pectoral fin development and morphogenesis of the apical ectodermal ridge in the killifish, Aphyosemiom scheeli. Anat. Rec. 204, 349-356.

Yamamoto, T. (1976). Series of stock culture in biological field. $I \prime$ "Medaka (Killifish), Biology and Strains." Keigaku Publishing Co., Tokyo, Japan. 